\title{
Identification of six serum antigens and autoantibodies for the detection of early stage epithelial ovarian carcinoma by bioinformatics analysis and liquid chip analysis
}

\author{
YUPENG ZOU* and $\mathrm{LI} \mathrm{LI}^{*}$ \\ Department of Gynecologic Oncology, Affiliated Tumor Hospital of Guangxi Medical University, \\ Nanning, Guangxi 530021, P.R. China
}

Received November 25, 2017; Accepted June 19, 2018

DOI: $10.3892 / \mathrm{ol} .2018 .9027$

\begin{abstract}
The early detection of ovarian cancer is critical for improving the prognosis of patients, but there are currently insufficient tumor biomarkers for early detection owing to their low diagnostic sensitivity and specificity. The aim of the present study was to investigate the use of the serum antigens $\mathrm{C}-\mathrm{C}$ motif chemokine ligand 18 and $\mathrm{C}-\mathrm{X}-\mathrm{C}$ motif chemokine ligand 1, and autoantibodies C1D, transmembrane $4 \mathrm{~L}$ six family member 1 , zinc finger protein 675 and fragile $X$ mental retardation 1 autosomal homolog 1 , for the early screening of epithelial ovarian cancer (EOC). The expression of these sex genes/proteins in ovarian cancer and normal ovarian tissue was examined, and the potential functions of the six genes/proteins in ovarian cancer were analyzed by bioinformatics. Finally, these data were verified in clinical samples, and the multi-analyte suspension array method was compared with the ELISA method. Taken together, these data indicated that these six genes/proteins may serve as potential biomarkers for the early detection of EOC.
\end{abstract}

\section{Introduction}

Ovarian cancer is the second most common tumor of the female reproductive system, with the highest mortality rate $(52.26 \%)$, United States in 2014 (1). The American Cancer Society estimates that 22,440 females in the USA were diagnosed with ovarian cancer and $\sim 14,080$ succumbed to the disease in 2017 (1). Epithelial ovarian cancer (EOC), one of the three major gynecological malignancy types, accounting for $85-90 \%$

Correspondence to: Professor Li Li, Department of Gynecologic Oncology, Affiliated Tumor Hospital of Guangxi Medical University, 71 Hedi Road, Nanning, Guangxi 530021, P.R. China E-mail: 1ili@gxmu.edu.cn

*Contributed equally

Key words: ovarian cancer, biomarker, early diagnosis, multi-index joint detection, liquid chip of malignant ovarian tumors and having the highest mortality rate (1). Due to the ovaries being deep in the pelvic cavity and there being no notable clinical symptoms in the early stages of ovarian cancer, the majority of patients with EOC have an advanced disease stage or distant metastasis at the time of diagnosis, resulting in limited treatment options and a 5-year survival rate of $<15 \%$ (2); therefore, it is vital to improve the rate of early detection. Currently, ovarian cancer is predominately diagnosed in clinical practice through pelvic examination, laparoscopy, the detection of serum cancer antigen 125 (CA125) (3), human epididymis secretory protein 4 (4) and other biomarkers, and imaging examination via computed tomography (CT), magnetic resonance imagining (MRI) and dynamic contrast enhanced (DCE)-MRI (5); however, the low diagnostic sensitivity and specificity of these methods has limited their efficacy in the early diagnosis of EOC. Thus far, there have been numerous studies regarding the diagnostic biomarkers of ovarian cancer $(3,4,6-8)$; however, there is not a suitable biomarker for the early diagnosis, treatment efficacy monitoring and prognosis determination of ovarian cancer. The study of Goff et al (6) demonstrated that the combination of multiple indicators for the diagnosis of ovarian cancer can not only improve the sensitivity and specificity of early diagnosis, but can also determine the selection of an effective treatment and prognosis.

In our preliminary study, surface-enhanced laser desorption/ionization-time of flight-mass spectrometry (SELDI-TOF-MS) technology was used to screen for the ovarian cancer diagnosis significance of the serum antigens C-C motif chemokine ligand 18 (CCL18) and C-X-C motif chemokine ligand 1 (CXCL1) (7). Serological analysis of recombinant cDNA expression library (SEREX) technology was simultaneously used to screen for serum autoantibodies, including C1D, transmembrane $4 \mathrm{~L}$ six family member 1 (TM4SF1), zinc finger protein 675 (TIZ) and fragile X mental retardation 1 autosomal homolog 1 (FXR1) (8). It was determined that CCL18 and CXCL1 had a high sensitivity for EOC diagnosis, but the specificity was not satisfactory; however, C1D, TM4SF1, TIZ and FXR1 had a high specificity in EOC diagnosis, compared with a combination of CCL18 and CXCL1. Therefore, it is hypothesized that the combined use of these different types of markers in the diagnosis of ovarian cancer may compensate for their respective disadvantages. 
In the present study, the expression of these six genes/proteins in normal ovarian and EOC tissues were initially analyzed using The Cancer Genome Atlas (TCGA) database. Secondly, the association between these six genes/proteins and their potential functions in EOC was analyzed. Finally, a mixed suspension of fluorescent microspheres was applied to prepare a liquid chip to combine these biomarkers for the differential diagnosis of EOC. In the present study, the objective was to combine the respective advantages of each set of biomarkers in order to minimize the limitations of a singular biomarker, for the early detection of EOC.

\section{Materials and methods}

Patients and samples. The present study was approved by the Ethics Committee of the Affiliated Tumor Hospital of Guangxi Medical University (Guangxi, China). All the patients provided written informed consent prior to sample collection. Serum specimens were collected from 60 patients diagnosed with early stage EOC, confirmed by pathological examination by a pathologist in the Affiliated Tumor Hospital of Guangxi Medical University, admitted between September 2003 and December 2012 to the Department of Gynecologic Oncology, Affiliated Tumor Hospital of Guangxi Medical University. Additionally, samples from 30 patients with gynecological benign tumors and 30 healthy females were collected following routine physical examinations between June and September 2008 (Table I). Early stage EOC was defined as stage I/II according to the International Federation of Gynecology and Obstetrics (FIGO, 2013) (9). Serum specimens were also collected from 323 patients (Table II) diagnosed by pathological examination admitted between September 2003 and October 2009 to the Department of Gynecologic Oncology in the Affiliated Tumor Hospital of Guangxi Medical University, including 119 patients with EOC with a median age of 48.5 years (range, 16-75 years) and 204 patients with benign pelvic tumors with a median age of 43.6 years (range, 15-59 years), as well as 120 healthy females, with samples collected from routine physical examinations in between June and September 2008, with a median age of 41.5 years (range, 21-70 years) (Table II). In addition, serum specimens were collected from 40 female patients with breast cancer (median age, 56.7 years; range, 20-75 years), 40 female patients with liver cancer (median age, 55.9 years; range, 38-87 years) and 40 female patients with lung cancer (median age, 54.3 years; range, 22-68 years). The Serum specimens were collected from the Affiliated Tumor Hospital of Guangxi Medical University between September 2003 and December 2012. Collected blood was stored at $4^{\circ} \mathrm{C}$ for $2 \mathrm{~h}$ until coagulated, prior to being centrifuged at 3,000 $\mathrm{x}$ g for $15 \mathrm{~min}$ at $4^{\circ} \mathrm{C}$, and the serum was stored at $-80^{\circ} \mathrm{C}$ until further use.

Non-ovarian cancer diseases were assessed in order to detect whether the diagnostic method has higher specificity for the diagnosis of ovarian cancer.

Bioinformatics analysis. The Human Protein Atlas (HPA; http://www.proteinatlas.org/) is an online tool with the aim of recording the distribution of all human proteins through the integration of various omics technologies, including antibody-based imaging, mass spectrometry-based proteomics, transcriptomics and systems biology. It consists of three separate parts, each focusing on a particular aspect of the genome-wide analysis of human proteins: The Tissue Atlas, which depicts the distribution of the proteins across all major tissues and organs in the human body; the Cell Atlas, which depicts the subcellular localization of proteins in single cells; and the Pathology Atlas, which depicts the impact of protein expression levels on the survival of patients with cancer. HPA was used to analyze the expression of C1D, CCL18, CXCL1, TM4SF1, FXR1 and TIZ in normal ovarian tissues and OC tissues, based on the clinical sample data from TCGA database (https://cancergenome.nih.gov/). A gene-gene/protein-gene/protein-protein interaction network was generated by GeneMANIA (http://genemania.org/). The Coremine Medical online tool (http://www.coremine. $\mathrm{com} /$ medical/) was used to annotate the associated biological processes.

The Genotype-Tissue Expression (GTEx) project collects and analyzes multiple human post mortem tissues. RNA-seq data from 31 of their tissues having a corresponding tissue in Human Protein Atlas were included to allow for comparisons between the Human Protein Atlas data and GTEx data. The GTEx RNA-seq data was mapped using the ensembl gene id available from GTEx, and the RPKMs (number Reads Per Kilobase gene model and Million mapped reads) for each gene were subsequently used to categorize the genes using the same classification as described above but using 0.5 RPKM as the threshold for detection.

Evaluating the diagnostic potential of C1D, CCL18, CXCL1, TM4SF1, FXR1 and TIZ in early stage EOC. As is subsequently described, C1D, CCL18, CXCL1, TM4SF1, FXR1 and TIZ pET-SUMO prokaryotic expression vectors were constructed in-house., and then purified high purity protein was produced. A method for the combined detection of CCL18, CXCL1, C1D, TM4SF1, FXR1 and TIZ was successfully developed using a multi-analyte suspension array (MASA). MASA was used to examine the C1D, CCL18, CXCL1, TM4SF1, FXR1 and TIZ expression in patients with EOC and gynecological benign tumors, and in healthy females.

The instrument used was a Bio-plex 200 suspension chip system as described below.

Establishment of a liquid suspension chip detection system. Subsequently, biotinylated antibody labeling was performed using an EZ-Link ${ }^{\circledR}$ Sulfo-NHS-LC-Biotinylation kit (cat. no. SF253102A; Thermo Fisher Scientific, Inc., Waltham, MA, USA), and the antibodies were measured using the bicinchoninic acid method (Pierce; Thermo Fisher Scientific, Inc.). In the second step, each antigen or antibody [Anti-GRO- $\alpha$ antibody (cat. no. ab89318; Abcam, Cambridge, UK), anti-macrophage inflammatory protein 4 antibody (cat. no. ab89338; Abcam), goat anti-human Immunoglobulin G Fc (cat. no. ab97221; Abcam) and natural human Immunoglobulin G protein (cat. no. ab91102; Abcam) was applied to carboxylated microspheres using the Bio-Plex ${ }^{\circledR}$ Amine Coupling Kit (cat. no. 171-406001; Bio-Rad Laboratories, Inc., Hercules, CA, USA). Finally, prepared samples of $1.25 \times 10^{6}$ microspheres were analyzed using the Bio-Plex Manager ${ }^{\mathrm{TM}}$ software (version 6.1; cat. 
Table I. Clinical pathological characteristics of the 60 patients with EOC, 30 with gynecological benign tumors and 30 healthy females.

\begin{tabular}{lccc}
\hline Variable & EOC $(\mathrm{n}=60)$ & Patients with gynecological benign tumors $(\mathrm{n}=30)$ & Healthy females $(\mathrm{n}=30)$ \\
\hline $\begin{array}{l}\text { Age, years } \\
\text { Mean }\end{array}$ & 46.7 & 32.4 & 34.4 \\
TNM stage (10) & 16 & 0 & 0 \\
$\begin{array}{l}\text { I-II } \\
\text { III-IV }\end{array}$ & 44 & 0 & 0 \\
OC type & $\begin{array}{l}\text { Serous cancer }(\mathrm{n}=42) \\
\text { Mucinous carcinoma (n=18) }\end{array}$ & Ovarian lutein cysts $(\mathrm{n}=3)$ & \\
& & Ovarian chocolate cysts $(\mathrm{n}=13)$ & \\
& & Ovarian mature teratoma $(\mathrm{n}=12)$ & \\
& Ovarian corpus luteum cyst $(\mathrm{n}=2)$ & \\
\end{tabular}

EOC, epithelial ovarian cancer; TNM, Tumor-Node-Metastasis.

Table II. Clinical characteristics of serum from patients.

\begin{tabular}{lr}
\hline Serum property & No. \\
\hline Ovarian Cancer & 119 \\
Epithelial tumor & 105 \\
Non-epithelial tumors & 14 \\
FIGO staging (10) & \\
I & 15 \\
II & 22 \\
III & 70 \\
IV & 12 \\
Pelvic benign tumor & 204 \\
Uterine-derived benign tumors & 57 \\
Tubal-derived benign tumors & 15 \\
Ovarian-derived benign tumors & 111 \\
Other sources of benign tumors & 21 \\
Healthy women & 120 \\
Breast cancer & 40 \\
Liver cancer & 40 \\
Lung cancer & 40
\end{tabular}

FIGO, International Federation of Gynecology and Obstetrics.

no. 171001010; Bio-Rad Laboratories, Inc.) for MASA chip detection.

ELISA detection of CID, TM4SF1, FXR1 and TIZ immunoglobulin $G$ autoantibodies. The levels of serum antigens CCL18 and CXCL1, and serum autoantibodies C1D, TM4SF1, FXR1 and TIZ were measured by an ELISA. All procedures were performed as described by the manufacturer's protocols for the ELISA kits. The ELISA test kits used were the Quantikine ${ }^{\circledR}$ ELISA Human CCL18/PARC Immunoassay kit (cat. no. DCL180B) and the Quantikine ELISA Human CXCL1/GRO $\alpha$ Immunoassay kit (cat.no. DGR00B) (both R\&D Systems, Inc., Minneapolis, MN, USA) and Rabbit anti-human
IgG (H+L), Biotin conjugated kit (cat. no. bsb-0297R) (Beijing Biosynthesis Biotechnology Co. Ltd., China).

Comparison of the MASA and ELISA methods. The accuracy, specificity and sensitivity between the MASA and ELISA methods in combined multi-index detection were compared. Firstly, the serum antigen and autoantibody contents were detected by the liquid suspension chip and ELISA methods, and the positive predictive value in the diagnosis of EOC was calculated by the liquid suspension chip and ELISA through logistic regression analysis and receiver operating characteristic (ROC) curves for the six indicators. The point of maximum diagnostic value, based on the Youden index, was defined as the diagnostic cut-off. Values above this cut-off were defined as positive, and values below the cut-off were defined as negative, If a value was equal to the cut-off, it was defined as negative. The number of positive and negative cases was counted, and the accuracy, specificity and sensitivity of multi-index combined detection was compared with the four-table method (Tables III and IV) (10).

Clinical evaluation of the multi-index detection of EOC. In the present study, a system was successfully established for the detection of the serum antigens CCL18 and CXCL1, and autoantibodies C1D, TM4SF1, FXR1 and TIZ, by liquid suspension microarray. The value and diagnostic performance of the combined detection of six indicators or CA125 in the diagnosis of early EOC was evaluated by testing the malignant and benign ovarian clinical samples. The logistic regression model was validated by detecting the levels of serum antigens CCL18 and CXCL1, and serum autoantibodies C1D, TM4SF1, FXR1 and TIZ in the MASA and ELISA. ROC curves were produced for the six indicators to determine their positive predictive value.

The efficacy of the six markers in the diagnosis of ovarian and non-ovarian malignancies was compared by measuring the relative content of the serum antigens CCL18 and CXCL1, and autoantibodies C1D, TM4SF1, FXR1 and TIZ, in the serum of 40 patients with breast cancer, 40 patients with liver cancer and 40 patients with lung cancer, using the liquid suspension chip method. The diagnostic value of the markers for ovarian cancer was compared with their value in other tumor types. 
Table III. Suspension liquid chip and ELISA method ovarian cancer detection accuracy.

\begin{tabular}{|c|c|c|c|c|c|c|c|}
\hline \multirow[b]{2}{*}{ Pathological diagnosis } & \multicolumn{3}{|c|}{ MASA } & \multirow[b]{2}{*}{ Pathological diagnosis } & \multicolumn{3}{|c|}{ ELISA } \\
\hline & Positive & Negative & Total & & Positive & Negative & Total \\
\hline Positive & 58 & 0 & 58 & Positive & 57 & 3 & 60 \\
\hline Negative & 2 & 60 & 62 & Negative & 3 & 58 & 61 \\
\hline Total & 60 & 60 & 120 & Total & 60 & 61 & 121 \\
\hline
\end{tabular}

Accuracy of liquid suspension chip, 98.33\%; accuracy of ELISA, 95.33\%; $\chi^{2}=39.31 ; \mathrm{P}=0.02$. MASA, multi-analyte suspension array.

Table IV. Sensitivity and specificity of liquid suspension chip and ELISA assay.

\begin{tabular}{|c|c|c|c|c|c|c|c|}
\hline \multirow[b]{2}{*}{ Pathological diagnosis } & \multicolumn{3}{|c|}{ MASA } & \multirow[b]{2}{*}{ Pathological diagnosis } & \multicolumn{3}{|c|}{ ELISA } \\
\hline & Positive & Negative & Total & & Positive & Negative & Total \\
\hline Positive & 58 & 0 & 58 & Positive & 57 & 3 & 60 \\
\hline Negative & 2 & 60 & 62 & Negative & 3 & 58 & 61 \\
\hline Total & 60 & 60 & 120 & Total & 60 & 61 & 121 \\
\hline
\end{tabular}

Sensitivity and specificity of liquid suspension chip, 100 and $96.7 \%$, respectively; sensitivity and specificity of ELISA, 96.1 and 95.1\%, respectively. MASA, multi-analyte suspension array.
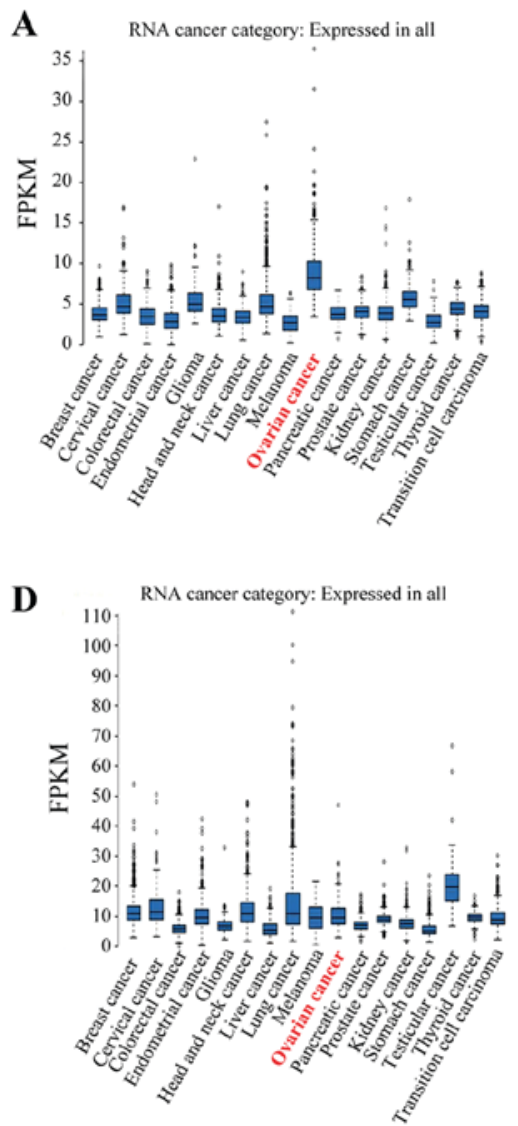
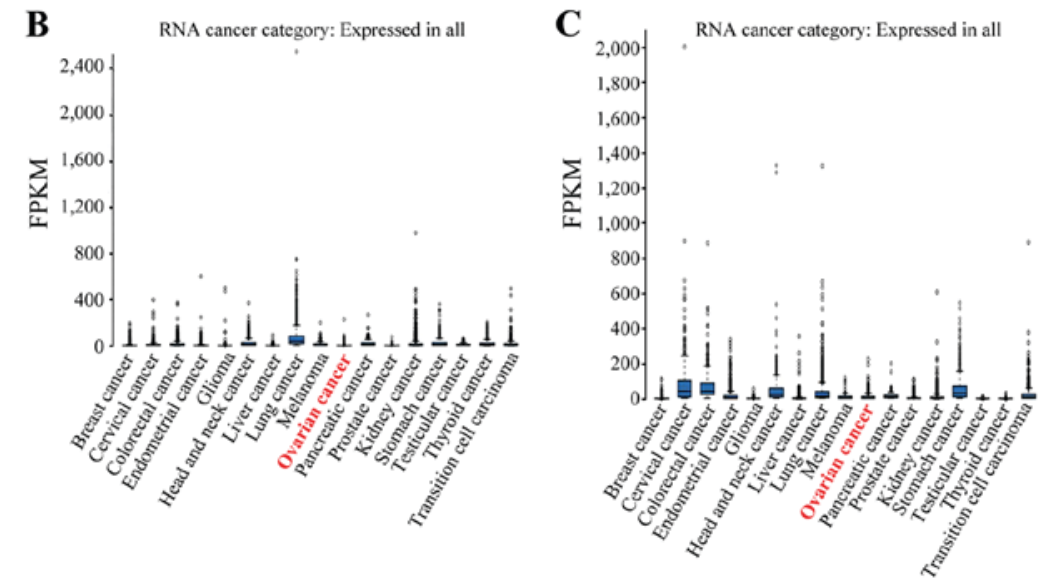

$\mathbf{E}$



$\mathbf{F}$

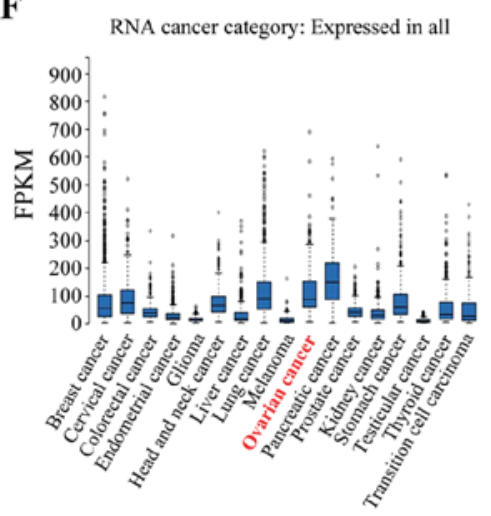

Figure 1. Overview of RNA-seq data from The Cancer Genome Atlas. The RNA-seq data of 17 cancer types are reported as the median FPKM. The RNA cancer tissue category, including cancer tissue enriched, cancer group enriched, cancer tissue enhanced, expressed in all, mixed and not detected, is calculated based on the mRNA expression levels across all 17 cancer tissue types. (A) C1D mRNA expression overview. (B) C-C motif chemokine ligand 18 mRNA expression overview. (C) C-X-C motif chemokine ligand 1 mRNA expression overview. (D) Fragile X mental retardation 1 autosomal homolog 1 mRNA expression overview. (E) Zinc finger protein 675 mRNA expression overview. (F) Transmembrane 4 L six family member 1 mRNA expression overview. FPKM, number of fragments per kb of exon per million reads. 

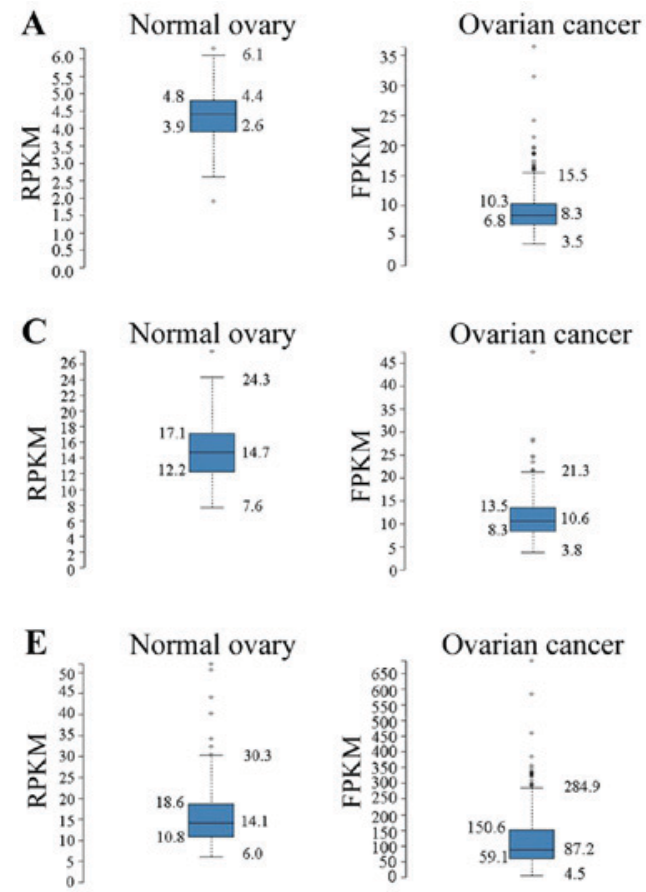


Figure 2. Ovarian RNA-seq data from the GTEx project 7 samples) and ovarian cancer RNA-seq data from TCGA (373 samples). The RNA-seq data are reported as the median number of RPKM for the GTEx data and as number of FPKM for TCGA data. The normalized distribution across the dataset is visualized with box plots, including the median, and 25th and 75th percentiles. Points are displayed as outliers if they are 1.5 -fold above or below the interquartile range. RPKM/FPKM values of the individual samples are presented next to the box plot. (A) Left panel, C1D expression in ovarian tissue based on GTEx RNA-seq data (mean RPKM, 4.4); and right panel, C1D expression in ovarian cancer tissue based on TCGA RNA-seq data (mean FPKM, 8.9). (B) Left panel, CXCL1 expression in ovarian tissue based on GTEx RNA-seq data (mean RPKM, 1.5); and right panel, CXCL1 expression in ovarian cancer based on TCGA RNA-seq data (mean FPKM, 12.4). (C) Left panel, FXR1 expression in ovarian tissue based on GTEx RNA-seq data (mean RPKM, 14.8); and right panel, FXR1 expression in ovarian cancer tissue based on TCGA RNA-seq data (mean FPKM, 11.2). (D) Left panel, TIZ expression in ovarian tissue based on GTEx RNA-seq data (mean RPKM, 1.1); and right panel, TIZ expression in ovarian cancer tissue based on TCGA RNA-seq data (mean FPKM, 2.0). (E) Left panel, TM4SF1 expression in ovary tissue based on GTEx RNA-seq data (mean RPKM, 16.7); and right panel, TM4SF1 expression in ovarian cancer based on TCGA RNA-seq data (mean FPKM, 114.7). (F) CCL18 expression in ovarian cancer tissue based on TCGA RNA-seq data (mean FPKM, 3.7). No GTEx RNA-seq data was identified for CCL18 in normal ovarian tissue. GTEx, Genotype-Tissue Expression; TCGA, The Cancer Genome Atlas; RKPM, reads per kb of exon per million reads; FPKM, fragments per kb of exon per million reads; CXCL1, C-X-C motif chemokine ligand 1; FXR1, fragile X mental retardation 1 autosomal homolog 1; TIZ, zinc finger protein 675; TM4SF1, transmembrane 4 L six family member 1; CCL18, C-C motif chemokine ligand 18.



Figure 3. Gene/protein interaction networks for C1D, CCL18, CXCL1, TM4SF1, FXR1 and TIZ, based on the GeneMANIA online tool. CXCL1, C-X-C motif chemokine ligand 1; FXR1, fragile X mental retardation 1 autosomal homolog 1; TIZ, zinc finger protein 675; TM4SF1, transmembrane 4 L six family member 1; CCL18, C-C motif chemokine ligand 18. 


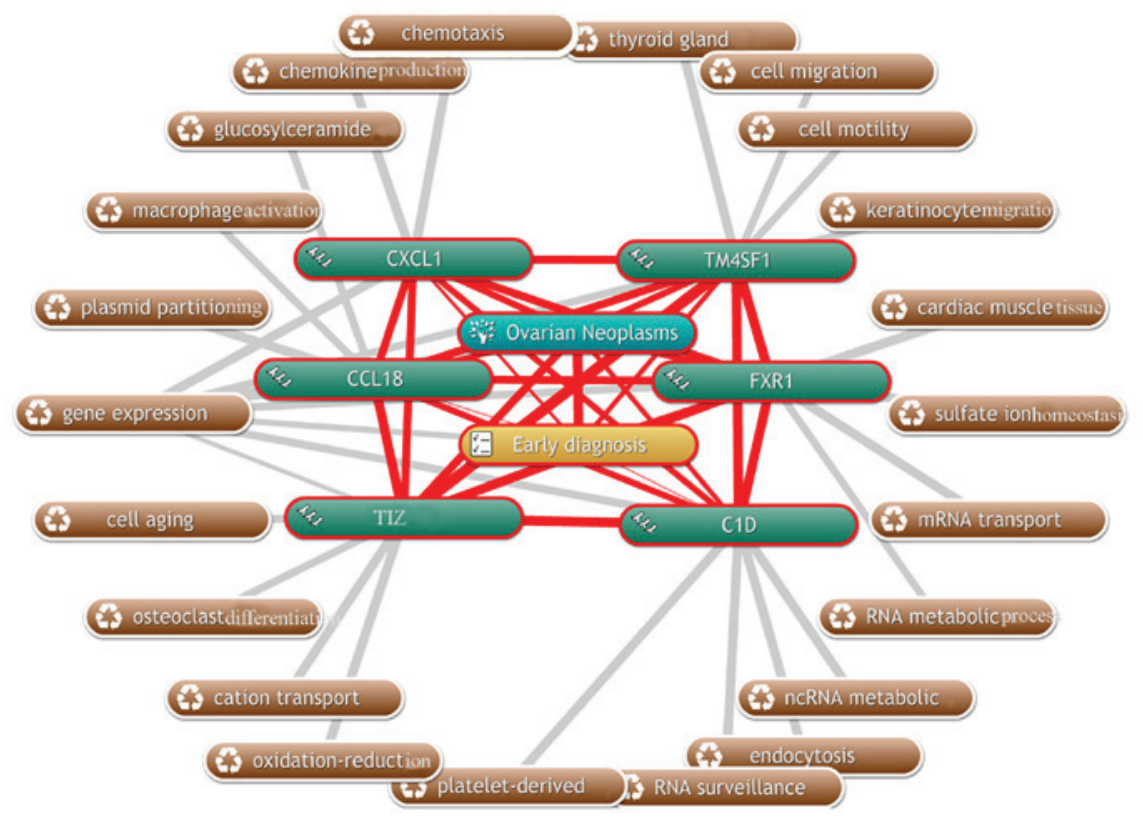

Figure 4. Diagram of the biological processes associated with C1D, CCL18, CXCL1, TM4SF1, FXR1 and TIZ (grey lines), and their association with early state ovarian cancer (red lines) using the Coremine Medical online tool. CXCL1, C-X-C motif chemokine ligand 1; FXR1, fragile X mental retardation 1 autosomal homolog 1; TIZ, zinc finger protein 675; TM4SF1, transmembrane $4 \mathrm{~L}$ six family member 1; CCL18, C-C motif chemokine ligand 18.
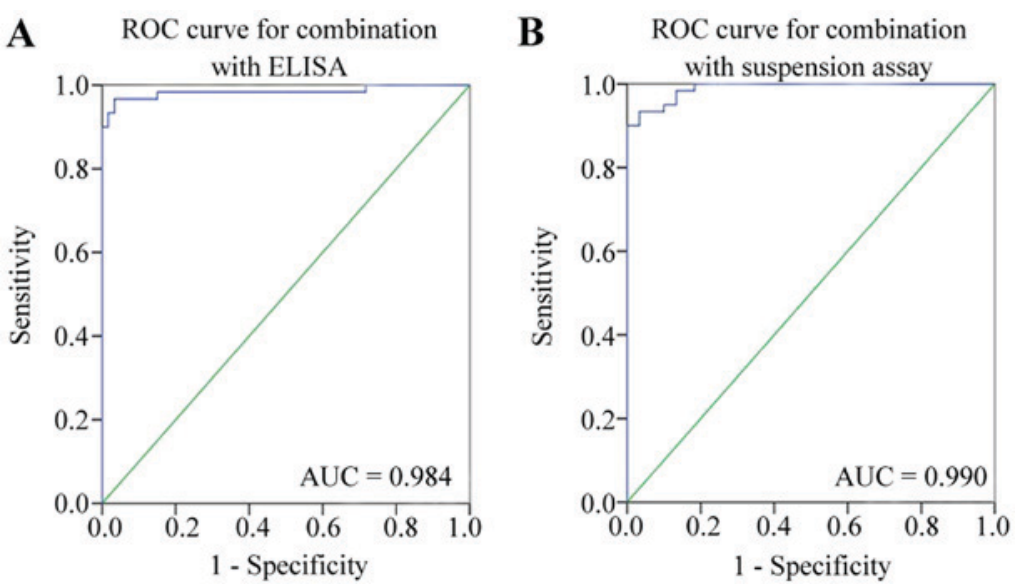

Figure 5. ROC curve analysis. ROC curves for the combination of the analysis of six proteins by (A) ELISA and (B) liquid suspension assay in the diagnosis of early stage epithelial ovarian cancer. AUC, area under the curve; ROC, receiver-operating characteristic.

ROC curves were produced for the six indicators to determine their positive predictive value.

Statistical analysis. SPSS statistical software (version 19.0; IBM Corp., Armonk, NY, USA) was used for all statistical analysis. Data are presented as the mean \pm standard deviation. Measurement data were analyzed by Student's t-test and $\chi^{2}$ test. Logistic regression analysis was used to draw the receiver operating curve (ROC curve). $\mathrm{P}<0.05$ was considered to indicate a statistically significant difference.

\section{Results}

Expression patterns of the six potential biomarkers in ovarian cancer. The expression of the six proteins in normal and tumor tissues was compared using the TCGA data. Their expression is associated with multiple tumor types, including ovarian cancer, and expression was notably lower in normal ovarian tissues, compared with ovarian cancer tissues $(\mathrm{P}<0.05$; Figs. 1 and 2).

Prediction and analysis of function based on gene/protein-gene/protein interactions. The interaction networks between C1D, CCL18, CXCL1, TM4SF1, FXR1 and TIZ and diagnosis-associated genes in EOC were analyzed using the GeneMANIA tool. The results demonstrated that C1D, CCL18, CXCL1, TM4SF1, FXR1 and TIZ interacted with 26 genes in total (Fig. 3).

Functional prediction based on the annotated biological processes. Coremine Medical is an online tool to identify the terms relevant to the biological function and importance in disease of genes and proteins. This tool was used with a P-threshold of $\mathrm{P}<1.000$, in order to associate CCL18, CXCL1, C1D, TM4SF1, TIZ and FXR1 with biological processes. As 
Table V. Diagnostic performance of the analysis of six proteins by liquid suspension assay and ELISA.

\begin{tabular}{|c|c|c|c|c|c|c|c|c|c|}
\hline $\begin{array}{l}\text { Detection } \\
\text { methods }\end{array}$ & $\begin{array}{l}\text { Accuracy } \\
(\%)\end{array}$ & $\begin{array}{c}\text { Sensitivity } \\
(\%)\end{array}$ & $\begin{array}{c}\text { Specificity } \\
(\%)\end{array}$ & LR+ & LR- & $\begin{array}{l}\text { Positive } \\
\text { predictive } \\
\text { value (\%) }\end{array}$ & $\begin{array}{l}\text { Negative } \\
\text { predictive } \\
\text { value }(\%)\end{array}$ & $\begin{array}{c}\text { Rate of } \\
\text { missed } \\
\text { diagnosis }(\%)\end{array}$ & $\begin{array}{c}\text { Rate of } \\
\text { misdiagnosis }(\%)\end{array}$ \\
\hline MASA & 98.33 & 96.67 & 100.00 & - & 0.03 & 100.00 & 96.77 & 3.33 & 0.00 \\
\hline ELISA & 95.04 & 95.00 & 95.08 & 19.32 & 0.05 & 95.00 & 95.08 & 5.00 & 4.92 \\
\hline
\end{tabular}

MASA, multi-analyte suspension array; LR, likelihood ratio.
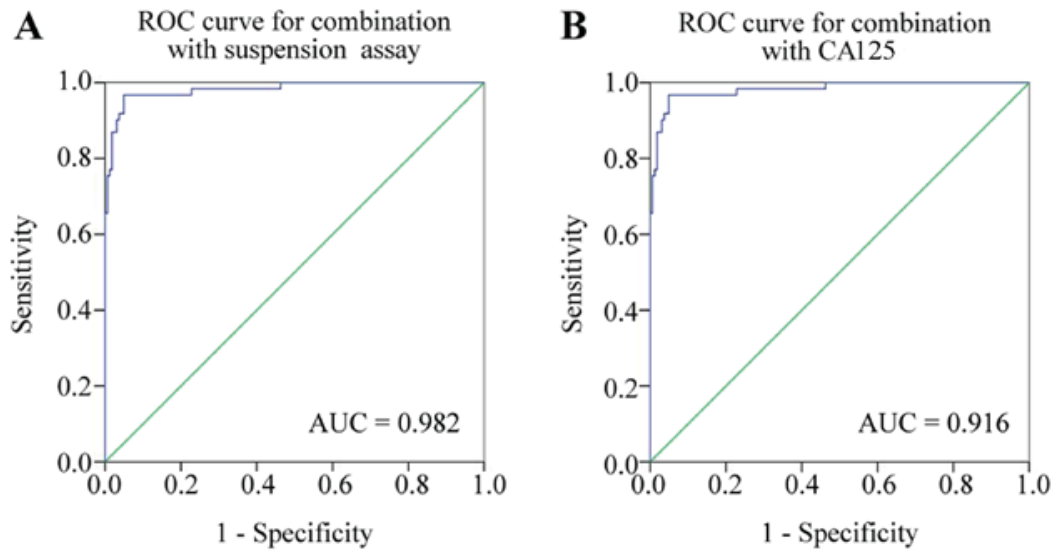

Figure 6. ROC curve analysis. ROC curves for the combination of the analysis of six proteins by (A) liquid suspension assay and (B) CA125 in the diagnosis of early stage epithelial ovarian cancer. AUC, area under the curve; ROC, receiver-operating characteristic; CA125, cancer antigen 125.

depicted in Fig. 4, 22 biological processes associated with early stage ovarian cancer and the expression levels of CCL18, CXCL1, C1D, TM4SF1, TIZ and FXR1 were annotated, indicating their mutual suitability for the early diagnosis of EOC. Among these, the association between the six proteins and early EOC is in accordance with the previous observation that CCL18, CXCL1, C1D, TM4SF1, TIZ and FXR1 were suitable biological markers for the early diagnosis of EOC.

Comparison of the accuracy of the liquid suspension chip and ELISA methods in multi-index combined detection. The areas under the ROC curves for the liquid suspension chip and the ELISA method detection of the six indexes for EOC diagnosis were 0.990 and 0.984 , respectively (Fig. 5). The accuracy of diagnosis using the liquid suspension chip and the ELISA method was 98.33 and $95.33 \%$, respectively (Table III). The differences between the methods was statistically significant $(\mathrm{P}=0.02)$. It was concluded that the diagnostic performance of the combined detected serum antigens CCL18 and CXCL1, and autoantibodies C1D, TM4SF1, FXR1 and TIZ was improved in terms of accuracy in the liquid suspension chip, compared with the ELISA method.

Comparison of sensitivity and specificity between the liquid suspension chip and ELISA methods. The sensitivity and specificity for the detection of the combination of the six genes by ELISA were 96.1 and $95.1 \%$, respectively, in the diagnosis of EOC (Table IV). The positive and negative likelihood ratio and predictive value, and the false positive and false negative rates for the liquid chip and ELISA methods are displayed in Table V. The diagnosis of EOC was improved in terms of sensitivity and specificity when using the suspension liquid chip, compared with the ELISA method.

Comparison of diagnostic value of the combination of the six proteins and CA125 in the diagnosis of EOC. Using a combination of the six indicators or CA125 to detect EOC resulted in an area under the ROC curve of 0.982 and 0.916 , respectively (Fig. 6). The positive and negative likelihood ratio and predictive value, and the false positive and false negative rates for the liquid chip combined detection and CA125 are depicted in Table VI. The diagnosis of EOC was improved when using the liquid chip, compared with CA125. It was concluded that the diagnostic performance of the combination of the serum antigens CCL18 and CXCL1, and autoantibodies C1D, TM4SF1, FXR1 and TIZ was improved when using the liquid suspension chip, compared with CA125.

Comparison of the efficacy of the six markers in the diagnosis of ovarian and non-ovarian malignancies. The positive rates for breast cancer, liver cancer, lung cancer and ovarian cancer based on the combined detection of the six indicators were $82.5,77.5,72.5$ and $94.9 \%$, respectively (Table VII), with an area under the ROC curve of $0.960,0.918,0.938$ and 0.983 , respectively (Fig. 7). The positive and negative likelihood ratio and predictive value, and the false positive and false negative rates for the diagnosis of these malignancies are displayed in Table VIII. It was concluded that the combined detection of the six indicators had a greater probability of indicating EOC, compared with the other malignancy types. 
Table VI. Diagnostic performance of the analysis of six proteins by liquid suspension assay and CA125.

\begin{tabular}{lccccccccc}
\hline Method & $\begin{array}{c}\text { Accuracy } \\
(\%)\end{array}$ & $\begin{array}{c}\text { Sensitivity } \\
(\%)\end{array}$ & $\begin{array}{c}\text { Specificity } \\
(\%)\end{array}$ & LR+ & LR- & $\begin{array}{c}\text { Positive } \\
\text { predictive } \\
\text { value }(\%)\end{array}$ & $\begin{array}{c}\text { Negative } \\
\text { predictive } \\
\text { value (\%) }\end{array}$ & $\begin{array}{c}\text { Rate of } \\
\text { missed } \\
\text { diagnosis (\%) }\end{array}$ & $\begin{array}{c}\text { Rate of } \\
\text { misdiagnosis (\%) }\end{array}$ \\
\hline MASA & 96.38 & 90.48 & 98.11 & 47.95 & 0.10 & 95.00 & 96.30 & 9.52 & 1.89 \\
CA125 & 81.08 & 60.47 & 94.12 & 10.28 & 0.42 & 86.67 & 79.01 & 39.53 & 5.88 \\
\hline
\end{tabular}

MASA, multi-analyte suspension array; CA125, cancer antigen 125; LR, likelihood ratio.

Table VII. Positive rates for other malignant tumor types detected by a suspension array combining six factors.

\begin{tabular}{lrcc}
\hline Sample category & $\mathrm{n}$ & Positive case & Positive rate $(\%)$ \\
\hline Ovarian cancer & 119 & 113 & 94.90 \\
Breast cancer & 40 & 33 & 82.50 \\
Liver cancer & 40 & 31 & 77.50 \\
Lung cancer & 40 & 29 & 72.50 \\
\hline
\end{tabular}

\section{Discussion}

The high mortality rate for ovarian cancer is primarily due to the predominately asymptomatic nature of the disease in its early stages; therefore, surgery and chemotherapy are the primary treatment choices for patients with ovarian cancer. Additionally, it is estimated that $70-75 \%$ of all females with ovarian cancer will experience recurrence, with a 5-year survival rate of $\sim 30 \%$ (11). When the disease can be detected in stage I and limited to the ovaries, up to $90 \%$ of patients can be successfully treated with the currently available surgical and chemotherapeutic methods. Early stage (I and II) ovarian cancer is only detected in a limited number of patients by conventional examination; therefore, a thorough understanding of the biomarkers for ovarian cancer identification and early detection may improve the 5-year survival rate (12).

TM4SF1 is overexpressed in EOC and regulates cancer cell motility and invasion. Additionally, it is associated with metastasis and regulates the development of angiogenesis, making it a potential target for anti-angiogenesis and antitumor therapies (13). TM4SF1 has been identified in human liver cancer (14), breast cancer (15), colorectal cancer (16) and ovarian cancer. Liu et al (17) identified that the positive expression rate of TM4SF1 protein in EOC tissue was higher than that in benign ovarian tumors or normal ovarian epithelial tissues, and may be associated with the abnormal proliferation of ovarian epithelial cells, malignant transformation and other clinical characteristics. TM4SF1 protein is associated with the occurrence and progression of EOC, but the underlying mechanism of this requires further study.

C1D is a small, 16-kDa mammalian nuclear matrix protein involved in higher-order chromatin folding and tight DNA binding. A recent study indicated that the C1D protein may be involved in the maintenance of genomic integrity by regulating the activity of double stranded DNA break repair proteins (18). C1D induces the production of autoantibodies in the serum
A

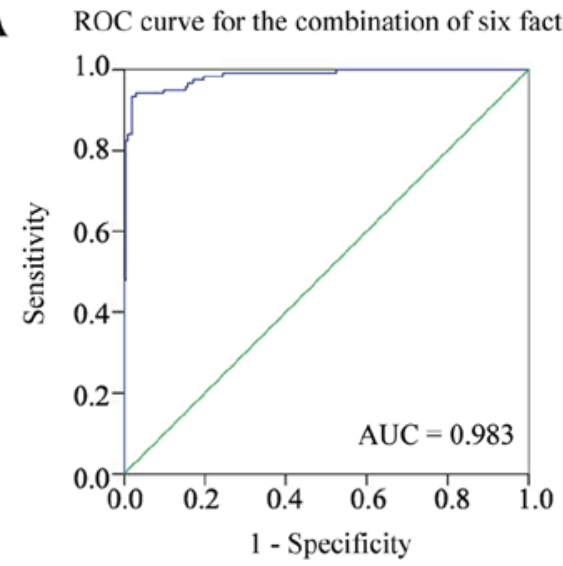

B ROC curve for the combination of six factors in the diagnosis of breast, liver or lung cancer

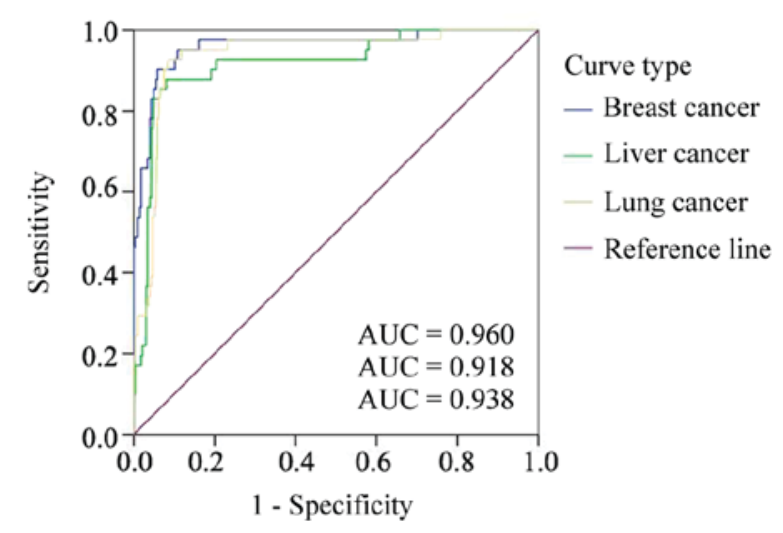

Figure 7. ROC curve analysis. ROC curves for the combination of the analysis of six proteins for the diagnosis of (A) epithelial ovarian and for (B) breast cancer, liver cancer and lung cancer. AUC, area under the curve; ROC, receiver-operating characteristic.

of patients with EOC, possibly due to the stimulation of the immune system by the ectopic expression of C1D (19); however, regarding the role of $\mathrm{C} 1 \mathrm{D}$ in the occurrence, development and apoptosis resistance of EOC, further research is required.

The FXR1 gene encodes an RNA-binding protein that is a critical regulator of post-transcriptional gene expression in differentiation, development and immunity. Due to FXR1 coordinating RNA-protein and protein-protein interaction networks, the altered function of FXR1 is expected to contribute toward the progression of cancer (20). In addition to its amplification in lung cancer, breast cancer, head and neck cancer and ovarian cancer $(21,22)$, FXR1 has been demonstrated to be downregulated in other human pathology types, 
Table VIII. Diagnostic performance of a suspension array combining six factors for other malignant tumor types.

\begin{tabular}{|c|c|c|c|c|c|c|c|c|}
\hline MASA & $\begin{array}{l}\text { Accuracy } \\
(\%)\end{array}$ & $\begin{array}{c}\text { Sensitivity } \\
(\%)\end{array}$ & $\begin{array}{l}\text { Specificity } \\
(\%)\end{array}$ & $\begin{array}{l}\text { Negative } \\
\text { predictive } \\
\text { value (\%) }\end{array}$ & LR+ & LR- & $\begin{array}{c}\text { Rate of } \\
\text { missed } \\
\text { diagnosis }(\%)\end{array}$ & $\begin{array}{c}\text { Rate of } \\
\text { misdiagnosis }(\%)\end{array}$ \\
\hline Ovarian cancer & 56.51 & 73.14 & 94.95 & 73.14 & 2.10 & 0.59 & 43.5 & 2.46 \\
\hline Breast cancer & 30.84 & 68.51 & 82.50 & 68.51 & 0.98 & 1.01 & 69.15 & 4.16 \\
\hline Liver cancer & 26.05 & 68.34 & 77.50 & 68.34 & 0.82 & 1.08 & 74.94 & 4.52 \\
\hline Lung cancer & 25.89 & 68.67 & 72.50 & 68.67 & 0.83 & 1.08 & 74.10 & 5.69 \\
\hline
\end{tabular}

MASA, multi-analyte suspension array; LR, likelihood ratio.

including facioscapulohumeral muscular dystrophy. The overexpression of FXR1 is associated with cell growth, migration and invasion, indicating that FXR1 has oncogenic activities. Anti-FXR1 autoantibodies can be detected in the serum of patients with EOC, but their function in the development of EOC requires further investigation (23).

TIZ belongs to the $\mathrm{C} 2 \mathrm{H} 2$-type zinc finger protein family, which can interact with tumor necrosis factor receptor-associated factor 6 , in order to serve a role in the regulation of the spread and migration of tumors (24). According to a previous clinical study, the expression level of TIZ protein in the serum of patients with malignant ovarian tumors was significantly higher, compared with patients with benign ovarian tumor and healthy individuals, which indicates a notable association between the expression level of TIZ and ovarian cancer (25).

CXCL1 belongs to the CXC chemokine family and is associated with cellular transformation, tumor growth and an increase in invasive potential (26). A previous study demonstrated that CXCL1 is associated with the occurrence, growth and metastasis of malignant tumors, and the overexpression of CXCL1 in EOC cells promoted proliferation, whereas CXCL1-silencing inhibited proliferation (27). The detection of serum CXCL1 provides a novel direction for the early diagnosis of EOC, but its clinical application requires further validation.

CCL18 is predominately produced by tumor-associated macrophages, and promotes the migration and invasion of breast cancer cells (28). It is expressed at higher levels in ovarian cancer ascites, compared with non-ovarian carcinomas. Its increased expression in OC tissue is associated with metastasis (29). The study of Schutyser et al (30) determined that patients with EOC and ascites had higher CCL18 expression levels than ovarian benign tumor patients with high immune staining in interstitial areas and a limited number of CCL18 antibodies. EOC cells exhibited positive CCL18 expression, and the relative expression level was higher than that in benign ovarian tumors and normal ovarian epithelial cells (31). CCL18 protein expression has potential as a novel tumor marker for EOC.

The detection of CCL18 and CXCL1 antigens has greater sensitivity for the diagnosis of EOC compared with CA125, but its specificity was not satisfactory, as the detection of CCL18 and CXCLL1 antigens had a poor diagnostic specificity for EOC; however, autoantibodies, including TM4SF1, have a high specificity for the diagnosis of EOC, but the diagnostic sensitivity was reduced, compared with CCL18. Liquid chips can contain multiple types of microspheres with different fluorescent dyes, combined with the corresponding serum antigens and antibodies in a covalent cross-linking manner. The analytes are mixed with the fluorescently encoded microspheres to form immune complexes, which can be detected through the two-laser detection of fluorescent signals, to simultaneously detect a number of different molecules in the same sample (32). The serum markers of EOC were screened by SELDI-TOF-MS and SEREX techniques and used to produce a liquid microarray. By combining these two different types of markers for the early diagnosis and differential diagnosis of ovarian cancer, their respective advantages can make up for the drawbacks of one marker type alone. The high-throughput, high-content liquid suspension chip method is highly applicable in clinical practice, representing the current direction of the development of serum molecular diagnostic technology. It also provides a method for the simultaneous detection of multiple types of markers.

In conclusion, based on a comprehensive bioinformatics analyses and MASA, the six indicators were identified to have an improved performance, compared with CA125, for the early diagnosis of EOC. The genes identified in the present study have the potential to improve the early diagnosis of EOC, although these possibilities require further research, including further validation with an increased number of clinical samples.

\section{Acknowledgements}

Not applicable.

\section{Funding}

The present study was supported by the Science and Technology Development Plan of Guangxi, China (grant no. 1140003A-33) and Science and Technology Development Plan of Guangxi, China (grant no. 1140003A-34).

\section{Availability of data and materials}

All data generated or analyzed during this study are included in this published article. 


\section{Authors' contributions}

Study development and design of methodology was the responsibility of LL, experiments were conducted and analyzed by YZ and LL. Application of statistical, mathematical, computation and presentation of the published work and revision and approval of the final manuscript was undertaken by $\mathrm{YZ}$ and LL.

\section{Ethics approval and consent to participate}

The present study was endorsed by the Ethics Committee of the Affiliated Tumor Hospital of Guangxi Medical University (Guangxi, China). All the patients provided written informed consent prior to sample collection.

\section{Patient consent for publication}

All study participants involved in this experiment provided consent to publish any relevant data or images.

\section{Competing interests}

The authors declare that they have no competing interests.

\section{References}

1. Siegel RL, Miller KD and Jemal A: Cancer statistics, 2017. CA Cancer J Clin 67: 7-30, 2017.

2. Sopik V, Iqbal J, Rosen B and Narod SA: Why have ovarian cancer mortality rates declined? Part II. Case-fatality. Gynecol Oncol 138: 750-756, 2015.

3. Abu Hassan SO, Nielsen DL, Tuxen MK, Petersen PH and Sölétormos G: Performance of seven criteria to assess CA125 increments among ovarian cancer patients monitored during first-line chemotherapy and the post-therapy follow-up period. Future Sci OA 3: FSO216, 2017.

4. Capriglione S, Luvero D, Plotti F, Terranova C, Montera R, Scaletta G, Schirò T, Rossini G, Benedetti Panici P and Angioli R: Ovarian cancer recurrence and early detection: May HE4 play a key role in this open challenge? A systematic review of literature. Med Oncol 34: 164, 2017.

5. Yuan SJ, Qiao TK, Qiang JW, Cai SQ and Li RK: The value of DCE-MRI in assessing histopathological and molecular biological features in induced rat epithelial ovarian carcinomas. J Ovarian Res 10: 65, 2017.

6. Goff BA, Agnew K, Neradilek MB, Gray HJ, Liao JB and Urban RR: Combining a symptom index, CA125 and HE4 (triple screen) to detect ovarian cancer in women with a pelvic mass. Gynecol Oncol 147: 291-295, 2017.

7. Wang Q, Zhang W, Li DR and Li L: Identification of two potencial serum biomarkers for ovarian cancer and clinical validation thereof. Zhonghua Yi Xue Za Zhi 88: 1012-1016, 2008 (In Chinese).

8. Yang ZJ, Yang G, Jiang YM, Ran YL, Yang ZH, Zhang W, Zhang JQ, Pan ZM and Li L: Screening and sero-immunoscreening of ovarian epithelial cancer associative antigens. Zhonghua Fu Chan Ke Za Zhi 42: 834-839, 2007.

9. Kandukuri SR and Rao J: FIGO 2013 staging system for ovarian cancer: What is new in comparison to the 1988 staging system? Curr Opin Obstet Gynecol 27: 48-52, 2015.

10. Zhao Y: Consistency test of the paired fourfold table. J Mathe Med 23: 386-387, 2010.

11. Han XR, Wen X, Li YY, Fan SH, Zhang ZF, Li H, Sun XF, Geng GQ, Sun S, Huang SQ, et al: Effect of different anesthetic methods on cellular immune functioning and the prognosis of patients with ovarian cancer undergoing oophorectomy. Biosci Rep 37: pii: BSR20170915, 2017.
12. Teng $\mathrm{C}$ and Zheng H: Low expression of microRNA-1908 predicts a poor prognosis for patients with ovarian cancer. Oncol Lett 14: 4277-4281, 2017.

13. Cao J, Yang JC, Ramachandran V, Arumugam T, Deng DF, $\mathrm{Li} \mathrm{ZS}, \mathrm{Xu} \mathrm{LM}$ and Logsdon CD: TM4SF1 regulates pancreatic cancer migration and invasion in vitro and in vivo. Cell Physiol Biochem 39: 740-750, 2016.

14. Huang YK, Fan XG and Qiu F: TM4SF1 promotes proliferation, invasion, and metastasis in human liver cancer cells. Int $\mathrm{J}$ Mol Sci 17: pii: E661, 2016

15. Sun Y, Xu Y, Xu J, Lu D and Wang J: Role of TM4SF1 in regulating breast cancer cell migration and apoptosis through PI3K/AKT/mTOR pathway. Int J Clin Exp Pathol 8: 9081-9088, 2015.

16. Park YR, Lee ST, Kim SL, Liu YC, Lee MR, Shin JH, Seo SY, Kim SH, Kim IH, Lee SO and Kim SW: MicroRNA-9 suppresses cell migration and invasion through downregulation of TM4SF1 in colorectal cancer. Int J Oncol 48: 2135-2143, 2016.

17. Liu H, Yan Y, Gao C and Yang Z: Expression and clinical significance of TM4SF1 protein in epithelial ovarian cancer. Chin J Oncol Prev Treat 6: 20-24, 2014 (In Chinese).

18. Jackson RA, Wu JS and Chen ES: C1D family proteins in coordinating RNA processing, chromosome condensation and DNA damage response. Cell Div 11: 2, 2016.

19. Li G, Liu J, Abu-Asab M, Masabumi S and Maru Y: XPB induces C1D expression to counteract UV-induced apoptosis. Mol Cancer Res 8: 885-895, 2010.

20. Raheja R and Gandhi R: FXR1: Linking cellular quiescence, immune genes and cancer. Cell Cycle 15: 2695-2696, 2016.

21. Majumder M, House R, Palanisamy N, Qie S, Day TA, Neskey D, Diehl JA and Palanisamy V: RNA-binding protein FXR1 regulates p21 and TERC RNA to bypass p53-mediated cellular senescence in OSCC. PLoS Genet 12: e1006306, 2016.

22. Qian J, Hassanein M, Hoeksema MD, Harris BK, Zou Y, Chen H, Lu P, Eisenberg R, Wang J, Espinosa A, et al: The RNA binding protein FXR1 is a new driver in the 3q26-29 amplicon and predicts poor prognosis in human cancers. Proc Natl Acad Sci USA 112: 3469-3474, 2015.

23. Jo YS, Kim SS, Kim MS, Yoo NJ and Lee SH: Frameshift mutation of FXR1 encoding a RNA-binding protein in gastric and colorectal cancers with microsatellite instability. Pathol Oncol Res 23: 453-454, 2017.

24. Bing-bing Z, Wei Z, Qi W, Zhi-jun Y and Li L: The effect of TIZ gene overexpression on biological characteristics of epithelial ovarian cancer cells. Tumor 32, 2012.

25. Zheng HY, Zheng HY, Zhou YT, Liu EL, Li J and Zhang YM: Changes of TIZ expression in epithelial ovarian cancer cells. Asian Pac J Trop Med 8: 157-161, 2015.

26. Wani N, Nasser MW, Ahirwar DK, Zhao H, Miao Z, Shilo K and Ganju RK: C-X-C motif chemokine 12/C-X-C chemokine receptor type 7 signaling regulates breast cancer growth and metastasis by modulating the tumor microenvironment. Breast Cancer Res 16: R54, 2014.

27. Sahingur SE and Yeudall WA: Chemokine function in periodontal disease and oral cavity cancer. Front Immunol 6: 214, 2015.

28. Li HY, Cui XY, Wu W, Yu FY, Yao HR, Liu Q, Song EW and Chen JQ: Pyk2 and Src mediate signaling to CCL18-induced breast cancer metastasis. J Cell Biochem 115: 596-603, 2014.

29. Wang Q, Tang Y, Yu H, Yin Q, Li M, Shi L, Zhang W, Li D and Li L: CCL18 from tumor-cells promotes epithelial ovarian cancer metastasis via mTOR signaling pathway. Mol Carcinog 55: $1688-1699,2016$

30. Schutyser E, Struyf S, Proost P, Opdenakker G, Laureys G, Verhasselt B, Peperstraete L, Van de Putte I, Saccani A, Allavena P, et al: Identification of biologically active chemokine isoforms from ascitic fluid and elevated levels of CCL18/pulmonary and activation-regulated chemokine in ovarian carcinoma. J Biol Chem 277: 24584-24593, 2002.

31. Wei Z, Ying-Zhu Y, Li L, Zhong-Mian P, Zhi-Jun Y and Qi W: Study on the localization and quantification of CCL18 protein in epithelial cells of ovarian cancer. J Guangxi Med Univ: 9-13, 2014.

32. Lin A, Salvador A and Carter JM: Multiplexed microsphere suspension array-based immunoassays. Methods Mol Biol 1318: 107-118, 2015. 